\title{
Numerical Investigation of Aerodynamic Characteristics by a Rotating Thin Plate
}

\author{
Yoshihiro Kubota ${ }^{*}$, Osamu Mochizuki² \\ ${ }^{1}$ Faculty of Science and Engineering, Toyo University, Kawagoe, Japan \\ ${ }^{2}$ Department of Biomedical Engineering, Toyo University, Kawagoe, Japan \\ Email: ${ }^{*}$ kubota548@toyo.jp
}

Received 4 March 2015; accepted 19 March 2015; published 23 March 2015

Copyright (C) 2015 by authorS and Scientific Research Publishing Inc.

This work is licensed under the Creative Commons Attribution International License (CC BY). http://creativecommons.org/licenses/by/4.0/

(c) (i) 0 pen Access

\begin{abstract}
In this study, we use a thin rotating plate to generate propulsion and lift for a paper plate. And the thin plate rotates along the spanwise axis. We numerically determine the influence on aerodynamic characteristics with a rotational velocity of the thin plate. The rotational velocity is obtained with spin parameter which is the ratio of the peripheral speed of the plate to the main flow velocity. And the numerical simulations based on the discrete vortex method show that the autorotation mode of the plate in a uniform flow appears naturally when the spin parameter is unity. Vortex formed from the backward-rotating edge is weaker than those generated from the forward-rotating edge of thin plate. The maximum lift generated at $S=0.75$ if $S<1$. The negative moment becomes negative for the nondimensional rotating speed $S \leq 1.75$. The most negative moment appears when $S=1$; at that time, autorotation occurs naturally.
\end{abstract}

\section{Keywords}

Autorotation, Thinplate, Vortex, Vortexmethod, Speed of Rotation, Spin Parameter

\section{Introduction}

The purpose of this study is to understand the aerodynamic characteristics to generate propulsion and lift for a thin rotating paper plate. The aerodynamics of freely falling paper plate such as business cards was studied to understand the fluttering or the tumbling. We are focusing on the aerodynamics characteristics of autorotation of thin paper plate. The autorotation is free to rotate with a fixed axis of a centroid of paper plate. The displacement of paper plate during the autorotation is not only vertical but also horizontal. Moreover, the horizontal displacement is not a swing motion. This means that the paper plate has the direction of displacement with a horizontal.

\footnotetext{
*Corresponding author.
} 
The paper plate during the autorotation generates the lift force [1]. So if we control the rotation of paper plate properly to generate the enough lift force during the autorotation, this might become a small flying plane such as a Micro Air Vehicle (MAV) Aono et al. (2011). This is a starting point of our study [2].

From the previous study, the relation between shape of plate and the role of vortex for thin rotating plate was studied by Kubota et al. [3]. They experimentally investigated the appropriate shape from the moment of inertia. The relation between the formation of lift force and the lateral vortex structure was also shown. The aerodynamics of freely falling paper plate was known with the fluttering, the tumbling, and the autorotation. Mittal et al. (2004) studied numerically about the flutter and tumble with a freely falling paper plate and the autorotation during paper in a uniform flow. Their result showed the effect of Reynolds number and relation between transient of fluttering to tumbling and thickness of paper. The instability on unsteady free fall of a thin solid object was computed by Bönisch et al. (2007) [4]. They computed the loci of unsteady free fall solid object during the fluttering and tumbling from the calculation of dynamics on slid body. Influence of density ration of paper and fluid was studied by Finn (2007) [5]. His numerical study showed that the changing of trajectory as tumbling, fluttering, and autorotation with a difference of density ratio. For the study of fluttering and tumbling, Andersen et al. (2005) showed the numerical study of relation between the inertia moment with geometry of paper plate and transient of fluttering to tumbling [6]. Also, they reported a comparison between numerical and experimental studies. This argued that the lift was dominated by the rotational velocity of plate. The experimental study for focusing on tumbling frequency was studied by Mahadevan et al. (1999) [7]. They concluded that the tumbling frequency was associated with the geometry of plate as the chord length of paper and the span length of paper. Hirata et al. (2011) reported the experimental study of aerodynamic characteristics of tumbling plate [8]. They were focusing on the inertia moment, rotation, lift coefficient, and drag coefficient of paper as aerodynamic characteristics. Their results proposed the empirical formulation of aerodynamic characteristics from a function of Reynolds number. Pesavento et al. (2004) were studied the aerodynamic lift during a tumbling [9]. The aerodynamic lift on a tumbling plate was dominated by the both falling velocity and angular velocity of stiff paper plate such as the business card. An influence of cross sectional geometry for freely falling paper was studied numerically by Jin et al. (2008) [10]. The influence of cross sectional geometry was the change of angular velocity. For a tumbling, an angular velocity dominated the lift force and this was studied by Pesavento et al. (2004) [9].

The main scope of our study is the generation of the lift force and the drag force due to the formation of vertex with the different rotational velocity of thin plate. The rotational velocity is obtained with the spin parameter which is the ratio of the peripheral speed of the plate to the main flow velocity.

In our previous study, we determined the aspect ratio of the plate by using a falling paper and observed that a plate with an aspect ratio of 7 achieved the longest flight distance, which meant it must have the highest lift/drag ratio. The numerical simulation allowed us to calculate the forces acting on the plate during rotation as a function of rotation speed. In addition, we discuss herein how to use a rotating plate to generate aerodynamic forces.

\section{Simulation Model}

We performed numerical simulations for nonsteady-state forces acting on the rotating plate. The phenomenon in a falling rectangular paper strip which rotates naturally along its spanwise axis is known as autorotation [1] [8]. The influence of peripheral speed of the plate relative to the main flow velocity was considered with spin parameter $S$, which is the ratio of the peripheral speed of the plate to the main flow velocity and is defined as follows [8]:

$$
S=\pi N c /(60 U)
$$

Here $N$ represents the revolution per minute of the rotating plate, $c$ is the plate chord length. Also, the Reynolds number $R e$ was used to determine the condition of flow. The rectangular plate with an aspect ratio 7 was used on the calculation, since the effective shape of plate was determined from our previous experimental investigation. Autorotation occurs at $S=1.0$ according to wind-tunnel tests at $R e=9 \times 10^{4}$ [11] and at $S=0.6$ according to experiments in air and water from $R e=1.79 \times 10^{3}$ to $1.23 \times 10^{4}$ [1] [12]. To compare the effects of the spin parameter on the flow field, we preformed numerical simulations for various $S$ values. The numerical simulations were performed using commercial software (VFS VOs-2D, College Master Hands Co.), which was based on the discrete vortex method. We investigated the vortex structure formed with rotating plate and found the effective rotating speed for a plate from the aerodynamic characteristics. 


\section{Results and Discussion}

From the observation on our previous study, vortex formed by the autorotating plate was acting on the generation of lift force. We simulated the flow by using a vortex-blob method to observe events near the plate during rotation. The numerical simulation was implemented with the discrete vortex method. A similar simulation was performed by lima, who simulated the flight of a butterfly by means of vortex blobs [13]. The result of our simulation for a half turn with $S=0.6$ is shown in Figure 1. Darker/red dots show clockwise (cw) vortex blobs, and brighter/blue dots show counter clockwise (ccw) vortex blobs. The vortex composed of cw vortex blobs, which is generated from the A edge on the figures, travels near the upper surface and induces a negative pressure on the upper wing surface, which produces lift. However, the vortex composed of ccw vortex blobs, which is generated from the B edge on the figures, offsets the strength of ccw vortex by interfering with the cw vortex. Thus, the effect of the ccw vortex on the surface pressure of the wing is small, even if the strength of the ccw vortex at the beginning is stronger than that of the cw vortex.

By numerical simulations, we also investigated the effects of the spin parameter $S$ on the aerodynamic characteristics. The calculated forces are shown in Figure 2 for different values of $S$. Averaged values of drag, lift, moment, and overall resultant force are denoted in the figure by $C_{\mathrm{x}}, C_{\mathrm{y}}, C_{\mathrm{m}}$, and $R$, respectively. In the range of $S$ that we tested, the magnitude of the resultant force is a maximum at $S=0.1$, and the extremum occurs from $S=$ 1.0 to 1.25. The resultant force is not always large even if $S$ is large. For example, the resultant force for $S=2$ is smaller than that for $S=1$. The force is a minimum at $S=0.6$ if $S<1$.

The drag shows tendencies which are similar to those of the resultant force. In contrast to the drag, the lift is a maximum at $S=2$ and decreases to almost zero at $S=1.25$ with decreasing $S$ in the range from $S=2$ to 1.25 . The extremum occurs at $S=0.75$ and becomes negative for $S \leq 0.25$. The sign of the lift changes at $S=0.4$, which indicates that the direction of the lift force is easily controlled by changing the rotation speed around $S=0.4$.

It is interesting that the $S$ of flapping insects is almost 0.5. For high maneuverability, we guess that insects evolved a ratio of flapping speed to flight speed that is near 0.4. The lift-generation mechanism for $S>1$ is the same as the Magnus effect for a rotating cylinder. However, for $S \leq 1$, lift is due to the difference of the pressures induced by vortices on both surfaces of the plate. The negative moment begins at the nondimensional rotation speed $S \leq 1.75$. The maximum value of the negative moment occurs at $S=1$; hence, autorotation occurs naturally. The moment of the force becomes negative except for $S=2$. Thus, the plate rotates automatically, because the negative moment assists rotation. Namely, it is called the autorotation mode. It appears naturally at $S=$ 1 because of the maximum absolute value of the negative moment.
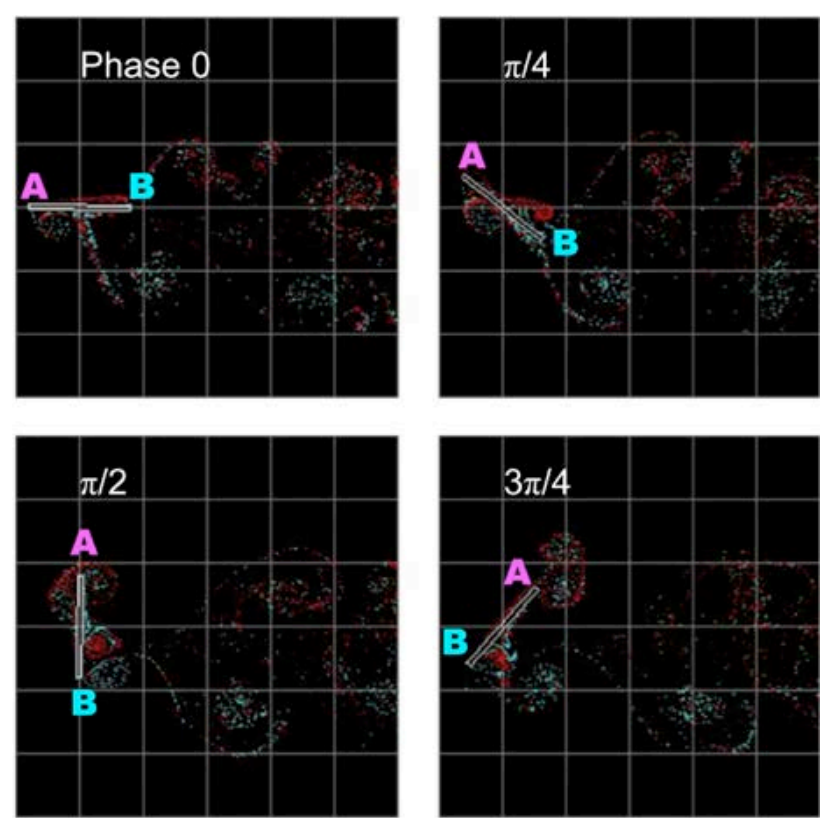

Figure 1. Process of vortex generation around plate rotating at $S=0.6$. Data obtained by numerical simulations. Darker dots show clockwise rotating vortex blobs, and brighter dots show counter clockwise rotating vortex blobs. 


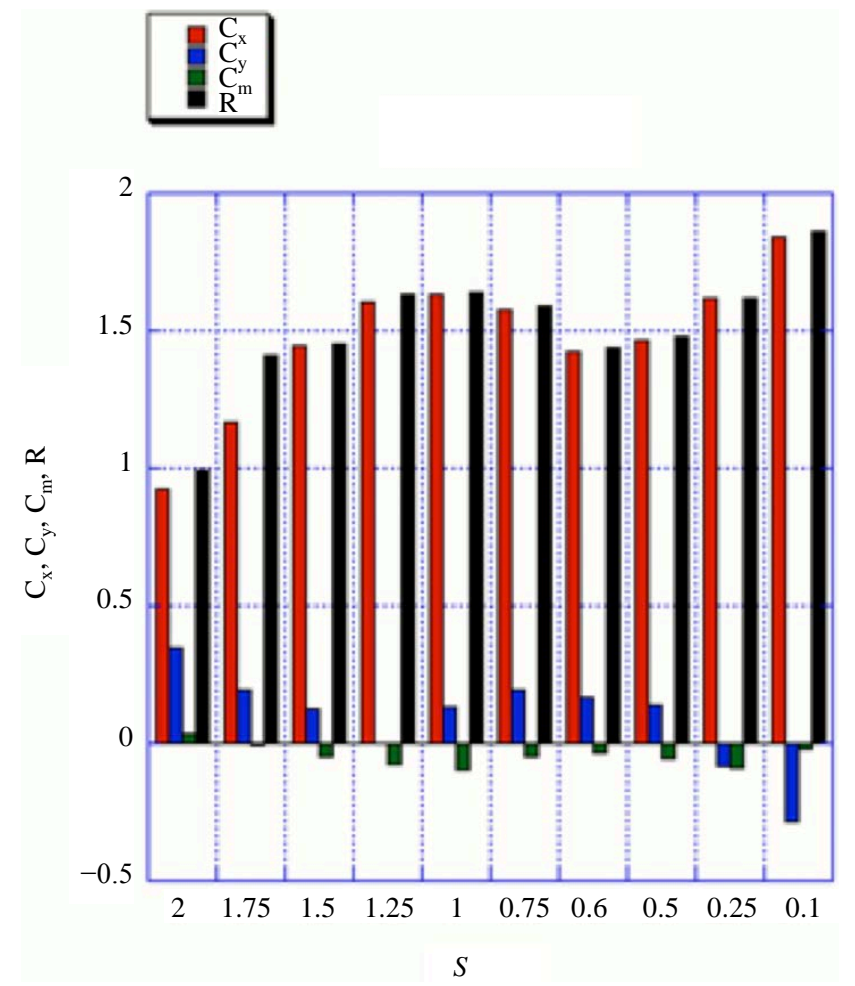

Figure 2. Relation between aerocynamic characteristics and rotationnal velocity. $C_{\mathrm{x}}$ : drag; $C_{\mathrm{y}}$ : lift; $C_{\mathrm{m}}$ : moment; $R$ : resulting overall force; and $S$ : spin parameter.

The ratio of lift to drag for different values of $S$ is shown in Figure 3. For $S=2$, which gives the largest ratio $C_{\mathrm{y}} / C_{\mathrm{x}}$, the flight distance in the forward direction is maximum. For $S=1.25$, which gives $C_{\mathrm{y}} / C_{\mathrm{x}}=0$, the plate falls vertically. The flight path never deflects, because it does not generate lift in this case. Moreover, for $S<1$, the maximum ratio $C_{\mathrm{y}} / C_{\mathrm{x}}$ appears at $\mathrm{S}=0.75$, and this ratio becomes increasingly negative at $S=0.1$ because of negative lift. A test plane with this rotating plate will fly backward.

Figure 4 shows Lissajous figures on the drag and lift in the horizontal and vertical axes, respectively. One loop corresponds to a half rotation of the plate. Thus, the results in each figure correspond to many rotations. Bundled lines show average change, and the width of these bundles shows fluctuations in each loop. Lissajous shows that the change of direction on force caused by the rotation of a thin plate. For $S=0.05$, approximately five peaks appear in a half rotation, which means that the force results from the combination of lift and drag points in five directions during a half rotation of the plate. Thus, the direction of the test plane will change five times per rotation if we choose this rotation speed, which means that we could not predict where it flies.

On the other hand, for $S=0.5-1.0$, the Lissajous loops are similar to each other. The size of the loop and the fluctuation become larger for $S$ increasing within the given range. For $S=1.25-2.0$, a protuberance appearing in the second quadrant grows with increasing $S$. The protuberance shows that negative drag (i.e., thrust) is generated at that phase. However, the drag is much larger than the thrust over a half rotation; therefore, we never notice the thrust generation at $S>1$ when we observe the average force. The larger fluctuation results from the changes in the overall force that is produced at larger $S$. Thus, according to the simulations, we should adopt $S=$ 1 for the effective rotating speed. We should make the plate easy to rotate so that an autorotation with $S=1$ is naturally selected.

\section{Conclusion}

We investigate the forces acting on the rotating plate with the vortex method. The influence of speed on rotation of plate is considered by using spin parameter $S$. Vortices generated from the backward-rotating edge are weaker than those generated from the forward-rotating edge. However, the former move near the wing surface together with the plate; therefore, the induced surface pressure is larger. The lift attains a maximum at $S=0.75$ if $S<1$. 


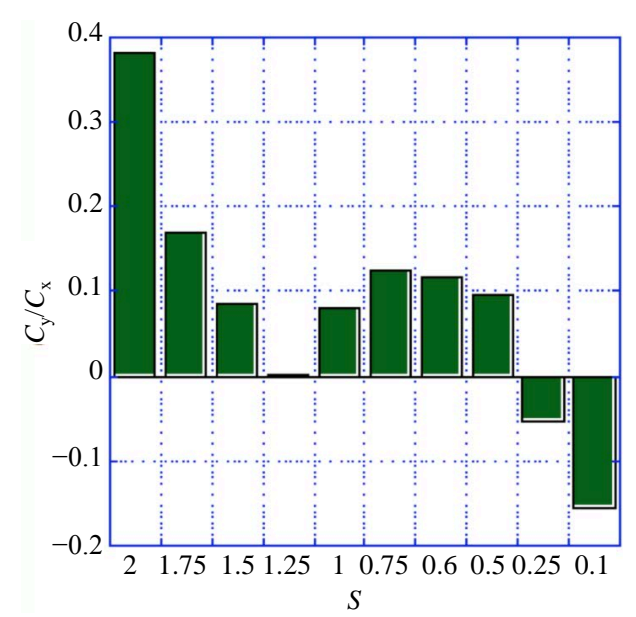

Figure 3. Ratio $C_{\mathrm{y}} / C_{\mathrm{x}}$ of lift to drag versus $S$.
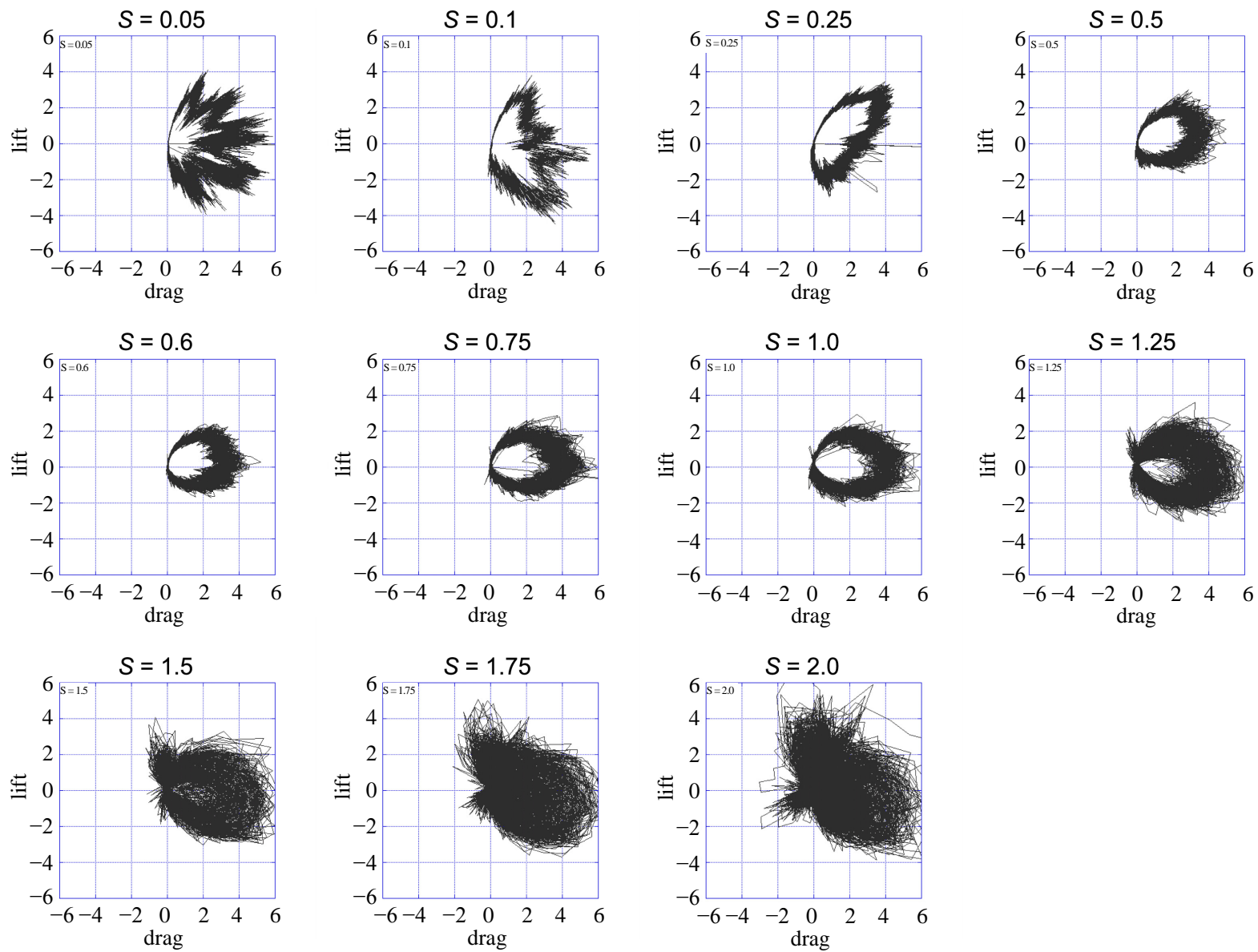

Figure 4. Lissajous figures for drag and lift with the drag on the horizontal axis and the lift on the vertical axis. Bundled lines show average change, and the width of these bundles shows fluctuations in each loop.

The moment becomes negative for the nondimensional rotating speed $S \leq 1.75$. The most negative moment occurs at $S=1$; thus, autorotation occurs naturally. The combination of lift and drag forces shows that the effective rotational speed for plate is $S=1$. Since, the slower rotational speed (i.e., $S=0.05$ ) causes the force generated unstably on the rotation cycle, and the rotating plate generates much larger drag than the thrust (or negative drag) during higher rotational speed (i.e., $S \geq 1.25$ ). 


\section{References}

[1] Mittal, R., Seshadri, V. and Udaykumar, H.S. (2004) Flutter, Tumble and Vortex Induced Autorotation. Theoretical and Computational Fluid Dynamics, 17, 165-170. http://dx.doi.org/10.1007/s00162-003-0101-5

[2] Aono, H., Liang, F.Y. and Liu, H. (2008) Near- and Far-Field Aerodynamics in Insect Hovering Flight: An Integrated Computational Study. The Journal of Experimental Biology, 211, 239-257. http://dx.doi.org/10.1242/jeb.008649

[3] Kubota, Y. and Mochizuki, O. (2013) Role on Moment of Inertia and Vortex Dynamics for a Thin Rotating Plate. World Journal of Mechanics, 3, 270-276. http://dx.doi.org/10.4236/wjm.2013.36028

[4] Bönisch, S. and Heuveline, V. (2007) On the Numerical Simulation of the Unsteady Free Fall of a Solid in a Fluid: I. The Newtonian Case. Computers and Fluids, 36, 1434-1445. http://dx.doi.org/10.1016/j.compfluid.2007.01.010

[5] Finn, D.L. (2007) Falling Paper and Flying Business Cards. SIAM News, 40.

[6] Andersen, A., Pesavento, U. and Wang, Z.J. (2005) Analysis of Transitions between Fluttering, Tumbling and Steady Descent of Falling Card. Journal of Fluid Mechanics, 541, 91-104. http://dx.doi.org/10.1017/S0022112005005847

[7] Mahadevan, L., Ryu, W.S. and Samuel, A.D.T. (1999) Tumbling Cards. Physics of Fluids, 11, 1-3. http://dx.doi.org/10.1063/1.869919

[8] Hirata, K., Hayakawa, M. and Funaki, J. (2011) On Tumbling of a Two-Dimensional Plate under Free Flight. Journal of Fluid Science and Technology, 6, 177-191. http://dx.doi.org/10.1299/jfst.6.177

[9] Pesavento, U. and Wang, Z.J. (2004) Falling Paper: Navier-Stokes Solutions, Model of Fluid Forces, and Center of Mass Elevation. Physical Review Letters, 93, 144501-1-144501-4. http://dx.doi.org/10.1103/PhysRevLett.93.144501

[10] Jin, C.Q. and Xu, K. (2008) Numerical Study of the Unsteady Aerodynamics of Freely Falling Plates. Communications in Computational Physics, 3, 834-851.

[11] Smith, E.H. (1971) Autorotating Wings: An Experimental Investigation. Journal of Fluid Mechanics, 50, 512-534. http://dx.doi.org/10.1017/S0022112071002738

[12] Kawaguchi, D., Ymauchi, K., Funaki, J. and Hirata, K. (2008) Free-Fall Experiments on a Tumbling Plate. JAXA-SP-08-009, 151-156 (in Japanese).

[13] Iima, M. (2007) A Two-Dimensional Aerodynamic Model of Freely Flying Insects. Journal of Theoretical Biology, 247, 657-671. http://dx.doi.org/10.1016/j.jtbi.2007.03.012

\section{Nomenclature}

$S$ : spin parameter, $\pi N_{c} /(60 U)$

$N$ : revolution per minute of the rotating plate; revolution/min.

$c$ : chord length of rotating plate; $\mathrm{mm}$

$U$ : main flow velocity; $\mathrm{m} / \mathrm{s}$

$C_{\mathrm{x}}$ : drag force acting on the rotating plate; $\mathrm{N}$

$C_{\mathrm{y}}$ : lift force acting on the rotating plate; $\mathrm{N}$

$C_{\mathrm{m}}$ : moment of force; $\mathrm{Nm}$

$R$ : overall resulting force; $\mathrm{N}$ 